\title{
An IR-UWB Photonic Distribution System
}

\author{
A. Kaszubowska-Anandarajah, Member, IEEE, P. Perry, Member, IEEE, L. P. Barry, Member, IEEE, and H. Shams
}

\begin{abstract}
Experimental results are presented for a novel distribution system for an impulse radio ultra-wideband (UWB) radio signals employing a gain-switched laser. The pulse position modulated short optical pulses with a bit rate of $1.25 \mathrm{~Gb} / \mathrm{s}$ are transmitted over fiber to a remote antenna unit, where the signal is converted to the electrical domain and undergoes spectral shaping to remove unwanted components according to UWB requirements. An experimental radio terminal has also been constructed to enable bit-error-rate measurements to be carried out. These experiments show that the optical distribution system will be capable of supporting the radio part of the system.
\end{abstract}

Index Terms-Gain-switched laser (GSL), pulse position modulation (PPM), radio-over-fiber (RoF), ultra-wideband (UWB) communications.

\section{INTRODUCTION}

$\mathbf{U}$ LTRA-WIDEBAND (UWB) communications systems have been developed for high bit rate wireless personal area networking applications. In some jurisdictions they can be deployed in the full 3.1- to $10.6-\mathrm{GHz}$ band but more typically use some sub-bands within that range [1]. To date, commercially available systems use a multicarrier orthogonal frequency-division-multiplexing [2] solution for bit rates of the order of $100 \mathrm{Mb} / \mathrm{s}$ with near term plans to extend this up to $480 \mathrm{Mb} / \mathrm{s}$. Such products are certified by the WiMedia Alliance and typically carry wireless universal serial bus certification.

This paper addresses a different approach which uses a pulsed modulation format generally known as impulse radio UWB (IRUWB) [3], which can deliver higher bit rates. It appears that the business case for using pulse-based schemes in the 3.1to $10.6-\mathrm{GHz}$ band is not currently viable as the cost/performance ratio is too high for current demand. It also appears to be technically challenging and expensive to use this band for high-definition multimedia interface applications operating at bit rates of up to $4.2 \mathrm{~Gb} / \mathrm{s}$ [4].

A new band, 57-64 GHz, is expected to become available for similar technology with the expectation that higher bit rates will be supported in this band [5]. Consequently, the possibility of using IR-UWB systems requires fresh investigation.

This paper presents a proof-of-concept experiment that shows the possible use of photonic techniques to generate optical pulse position modulation (PPM) signals that can be

Manuscript received April 08, 2008; revised July 29, 2008. First published September 3, 2008; current version published October 31, 2008. This work was supported by the Science Foundation Ireland Research Frontier Grant.

The authors are with the Radio and Optical Communication Laboratory, Research Institute for Networks and Communications Engineering, Dublin City University, Glasnevin, Dublin 9, Ireland (e-mail: Aleksandra.kaszubowska@dcu.ie).

Color versions of one or more of the figures in this letter are available online at http://ieeexplore.ieee.org.

Digital Object Identifier 10.1109/LPT.2008.2004989

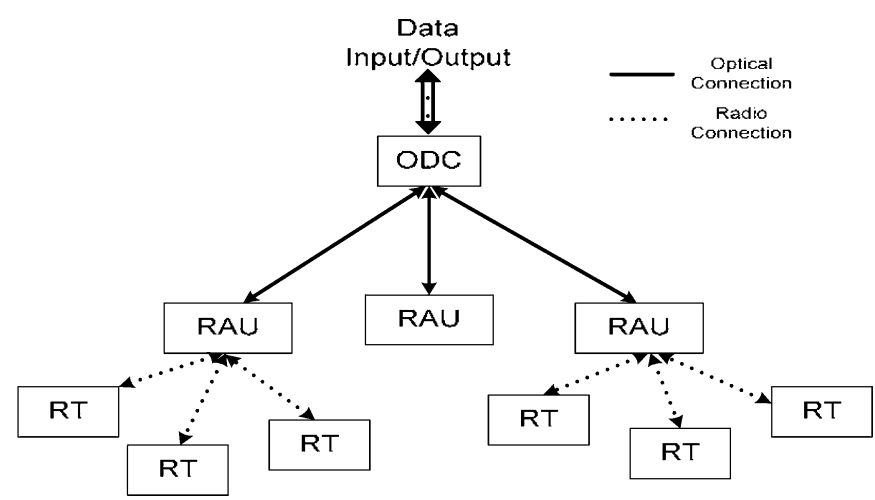

Fig. 1. System architecture.

distributed through a building and easily converted to UWB radio signals at remote antenna units (RAUs) to create a patchwork of UWB coverage areas that is analogous to a femto-cellular radio system.

This distribution system is centered on the internet access point to the building, for example, a residential broadband gateway that typically manages both internet access and voice-over-internet protocol telephony. In the case of triple-play deployments, the gateway also manages video distribution to the home and may handle up to $1 \mathrm{~Gb} / \mathrm{s}$ at present. Such a system takes advantage of the increased reach afforded by the use of fiber distribution within the building rather than a single radio hub. It also enables the distribution of multiple streams of high-definition television (HDTV) signals within the home from a centralized source to multiple receivers. This scenario then, requires extremely high bit rates to support HDTV distribution to the receivers, but relatively low bit rates for the reverse channel. The concept of IR-UWB has attracted particular attention recently from the photonics community with a view to satisfying the above scenario [6], [7].

\section{EXPERIMENTAL SETUP}

The experiment described here includes new approaches to pulse generation and distribution that offers considerable cost benefit compared to the regular radio-over-fiber (RoF) approach which requires a high-performance laser component. The system shown in Fig. 1 is divided into three subunits, as follows:

1) optical distribution center (ODC);

2) remote antenna unit (RAU);

3) radio terminal (RT).

In this proof-of-concept experiment, a single down link path from the ODC to an RAU and a single RT is investigated. The setup in Fig. 2 shows the equipment used in the ODC for this experiment, although a commercial deployment would rely on digital signal processing techniques to simplify the hardware 


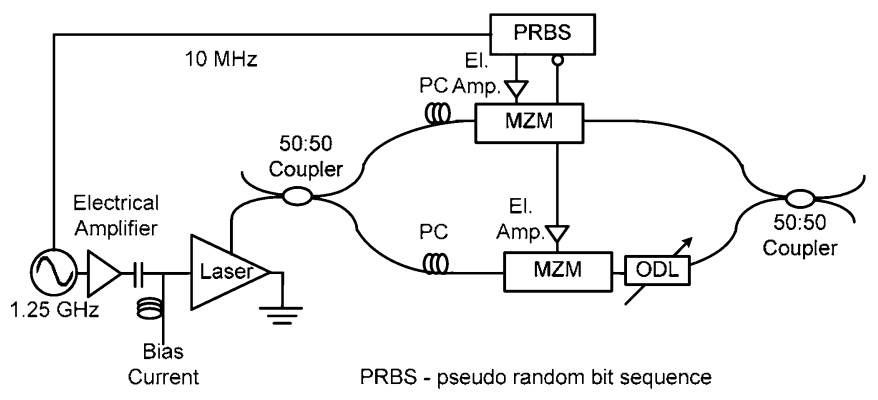

Fig. 2. ODC setup.

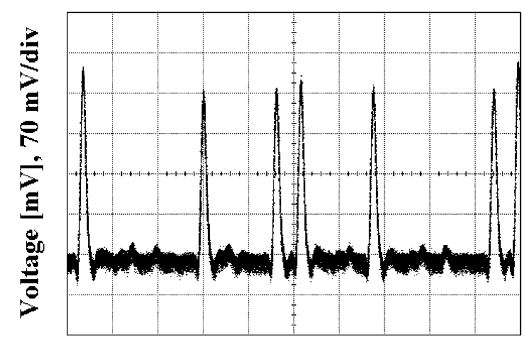

Time [ps], $500 \mathrm{ps} / \mathrm{div}$

Fig. 3. PPM modulated optical pulses (data sequence: 1001101).

used and for clock recovery rather than the simple hardwired solution used here. It consists of a gain-switched laser (GSL) [8] that is driven from a sinusoidal RF generator at the clock frequency. The resulting pulses were approximately $70 \mathrm{ps}$ wide and were fed to a pair of Mach-Zehnder modulators (MZMs). The data stream was generated with a 50:50 mark space ratio with sufficient amplitude to drive the MZMs with both data and inverted data so that one MZM passed a pulse in the presence of a logical "1" and the other MZM passed a pulse in the presence of a logical " 0. ." The pulse pattern generator and the signal generator, used for gain switching of the laser, were synchronized using the $10-\mathrm{MHz}$ reference signal. It should be noted that accurate phase adjustments are required to align the optical impulse with the center of the bit period. An optical delay line was used to delay the pulses from the lower MZM before recombining them, so that a PPM signal is created (as seen in Fig. 3). It should be noted here that the pulse positions are fixed within the symbol period, rather than the time hopping IR-UWB systems that might be realistically deployed [9]. Applying time hopping is expected to improve the spectral flatness and reduce the peak to average power.

The RAU in this experiment (Fig. 4) was simply a photodetector and an electrical bandpass filter (BPF). The RF spectrum of the detected optical pulses directly after the electrical amplifier is shown in Fig. 5(a). It can be seen that the signal does not comply with the Federal Communications Commission (FCC) spectral mask and it includes both low (dc to $2.5 \mathrm{GHz}$ ) and high (above $10 \mathrm{GHz}$ ) frequency components. This means that the signal contains sufficiently strong spectral components to fill the UWB spectrum, but electrical filtering is clearly required. Fig. 5(b) shows the RF spectrum of the signal after the BPF which has a pass-band of 3.1 to $10.6 \mathrm{GHz}$ that was designed to shape the spectrum to the FCC mask for UWB

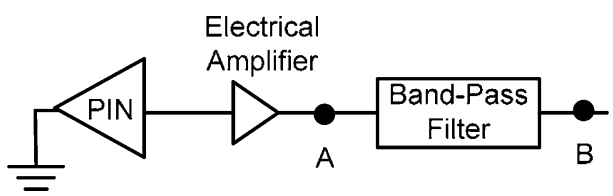

Fig. 4. RAU.

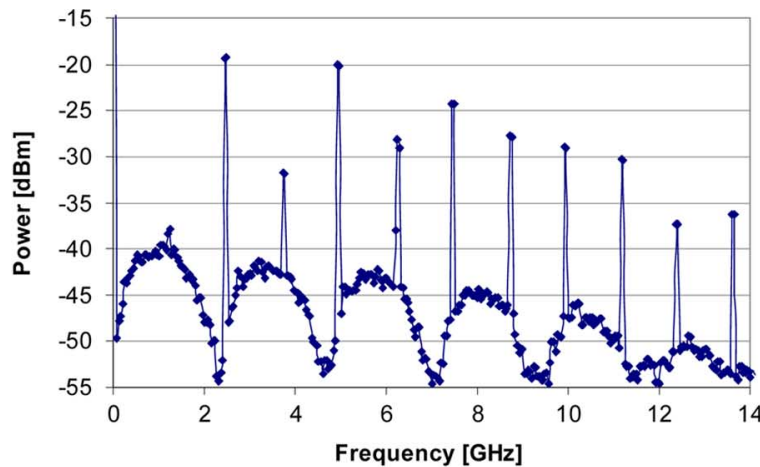

(a)

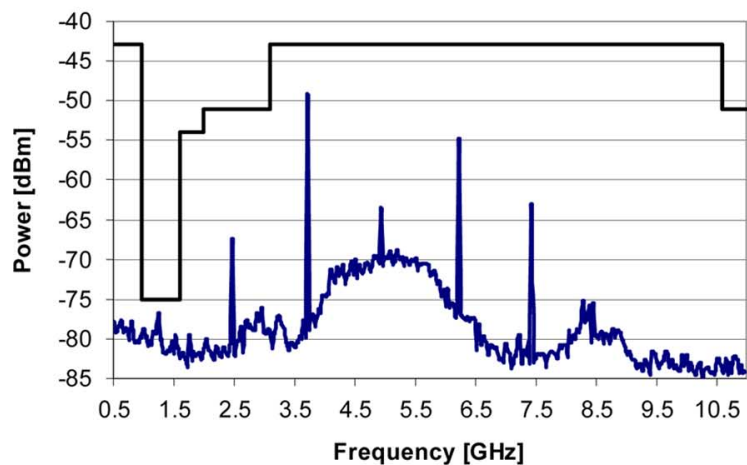

(b)

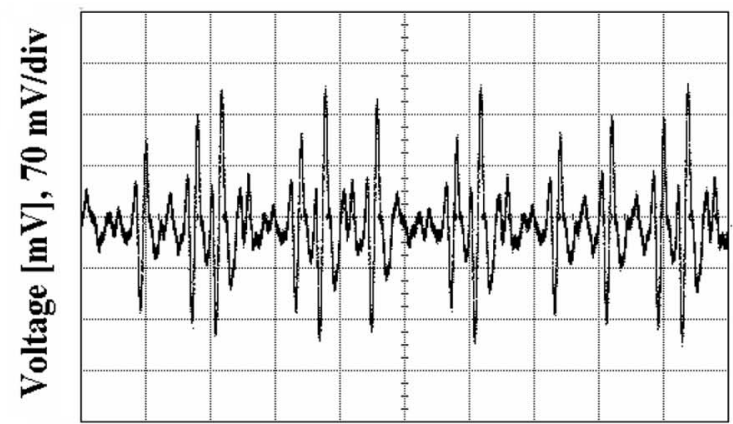

(c)

Time [ns], 1 ns/div

Fig. 5. (a) RF spectrum of the detected optical pulses (point A in Fig. 4); (b) RF spectrum after the BPF and the FCC spectrum mask for indoor communication; (c) temporal waveform of the UWB signal (point B in Fig. 4); data sequence: 001011010001)

transmissions [10]. The BPF has a double pi topology that has been designed in-house using standard techniques [11] and implemented using both distributed elements and lumped capacitors yielding a group delay ripple of $230 \mathrm{ps}$. It can be seen that the spectral components below $3 \mathrm{GHz}$ have been greatly reduced, and that the signal after filtering satisfies the stringent requirements of the FCC mask for UWB indoor emissions. Fig. 5(c) displays the temporal waveform of the $1.25-\mathrm{Gb} / \mathrm{s}$ UWB signal recorded at point B in Fig. 4. Even though the intersymbol interference is apparent in the plot, the data sequence (001011010001) can still be recognized. In the complete system 


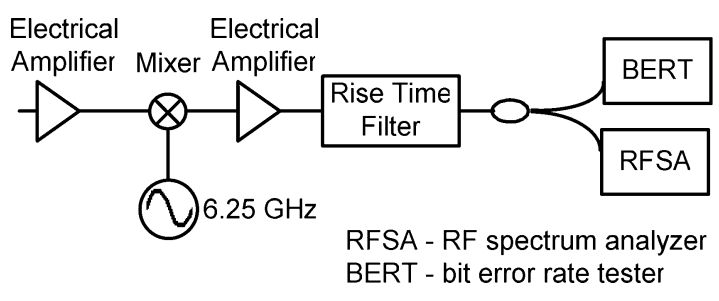

Fig. 6. RT setup.

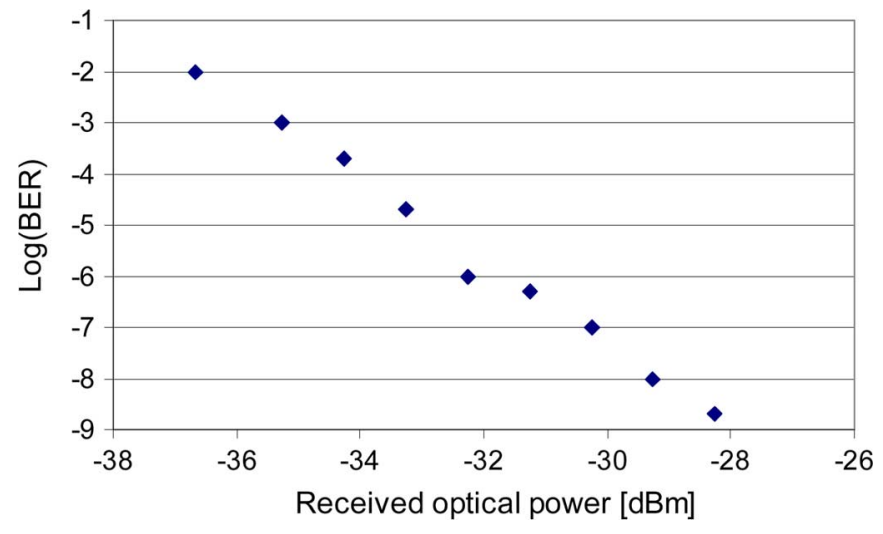

Fig. 7. BER versus received optical power at RAU.

this signal would be then transmitted though the radio channel to the RT. However, in our experiment no radio transmission is realized, therefore the output of the BPF is connected directly to the RT.

The RT for this experiment was composed of an amplifier with 30-dB gain driving a high bandwidth mixer and is shown in Fig. 6. The mixer received a 7-dBm local oscillator signal at $6.25 \mathrm{GHz}$ that was frequency locked to the data clock in the ODC and had manual phase shifting capability. The intermediate frequency (IF) output, therefore, contained the fifth harmonic of the data signal down-converted to dc, with the upper and lower sideband at 5 and $7.5 \mathrm{GHz}$ being down-converted to the baseband data rate of $1.25 \mathrm{GHz}$. The IF signal was then passed through a low-pass filter with a corner frequency of $746 \mathrm{MHz}$ to remove additional harmonics and convert the data from return-to-zero format to nonreturn-to-zero format. The eye diagram of the down-converted signal is shown in Fig. 7 (optical power incident on the detector at RAU is $-28 \mathrm{dBm}$ ). This output was then analyzed with a bit-error-rate (BER) tester and an oscilloscope. The measured BER versus optical power is shown in Fig. 8. From the plot it can be seen that the optical distribution system is highly efficient, ensuring an error-free performance of the system at power levels as low as $-28 \mathrm{dBm}$. This means that it can operate without the need for optical amplification in residential and small office scenarios. Furthermore, with a higher power pulse source, the system can be overlaid on an existing passive optical network (PON) [12] for large-scale deployments.

\section{CONCLUSION}

A novel system design of an IR-UWB RoF system based on a GSL has been proposed and demonstrated. This method uses a

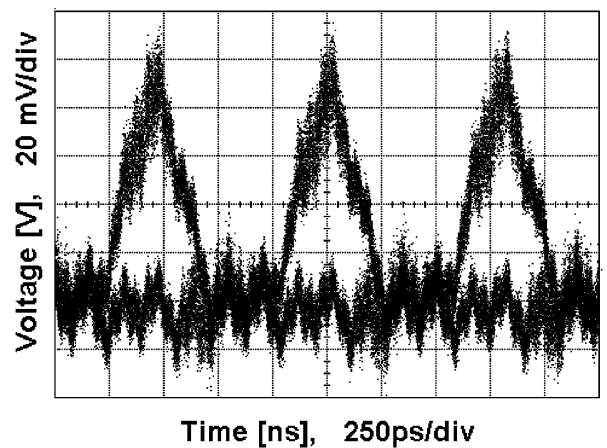

Fig. 8. Received eye diagram of the down-converted signal.

single laser and additional components that lend themselves to integration, thus offering the possibility of a low-cost and reliable UWB distribution system for residential or small office deployments. Our results show that optical pulses generated using the GSL can be easily converted to electrical monocycles fulfilling the FCC spectral requirements by the use of simple electrical BPF. Furthermore, we have demonstrated that this method allows for a reliable signal transmission and detection at power levels as low as $-28 \mathrm{dBm}$. This high power margin would allow the system to utilize the existing PON networks with high splitting ratio to provide large coverage areas.

\section{REFERENCES}

[1] Y. Le Guennec, M. Lourdiane, B. Cabon, G. Maury, and P. Lombard, "Technologies for UWB-over-fiber," in Special Symp. Photonic Generation and Distribution of Ultra-Wideband Signals, LEOS Annual Meeting, Montreal, Canada, 2006.

[2] Y. Zhang and J. Zhao, "Performance simulation of fixed-point for MB-OFDM UWB system," in Proc. Int. Conf. Wireless Communications, Networking and Mobile Computing, 2005, pp. 292-295.

[3] R. Llorente, T. Alves, M. Morant, M. Beltran, J. Perez, A. Cartaxo, and J. Marti, "Optical distribution of OFDM and impulse-radio UWB in FTTH networks," in Proc. OFC/NFOEC, San Diego, CA, 2008, Paper JWA109.

[4] R. Krishnamoorthy, "High definition, anywhere: How ultra wideband makes wireless HDMI possible," in 4th IEEE Consumer Communications and Networking Conf., Jan. 2007, pp. 395-399.

[5] C. Snow, "Ultra wideband communications past, present and future," in 2nd UBC-IEEE Workshop on Future Communications Systems, Vancouver, Canada, Mar. 9, 2007.

[6] A. M. J. Koonen, M. G. Larrode, A. Ng'Oma, K. Wang, H. Yang, Y. Zheng, and E. Tangdiongga, "Perspectives of radio over fibre technologies," in OFC/NFOEC, San Diego, CA, 2008, Paper OThP3,

[7] C. Wang, F. Zeng, and J. P. Yao, "All-fiber ultra wideband pulse generation based on spectral shaping and dispersion-induced frequency-to-time conversion," IEEE Photon. Technol. Lett., vol. 19, no. 3, pp. 137-139, Feb. 1, 2007.

[8] P. Anandarajah, P. Maguire, A. Clarke, and L. Barry, "Self-seeding of a gain-switched integrated dual-laser source for the generation of highly wavelength-tunable picosecond optical pulses," IEEE Photon. Technol. Lett., vol. 16, no. 2, pp. 629-631, Feb. 2004.

[9] J. Mitra and L. Lampe, "Robust detectors for TH IR-UWB systems with multiuser interference," in IEEE Int. Conf. Ultra-Wideband (ICUWB 2007), Sep. 2007, pp. 745-750.

[10] D. Porcino and W. Hirt, "Ultra-wideband radio technology: Potential and challenges ahead," IEEE Commun. Mag., vol. 41, no. 7, pp. 66-74, Jul. 2003.

[11] , E. H. Fooks and R. A. Zakarevicius, Eds., Microwave Engineering Using Microstrip Circuits. Englewood Cliffs, NJ: Prentice-Hall, 1990.

[12] M. D. Vaughn, D. Kozischek, D. Meis, A. Boskovic, and R. E. Wagner, "Value of reach-and-split ratio increase in FTTH access networks," $J$. Lightw. Technol., vol. 22, no. 11, pp. 2617-2622, Nov. 2004. 